\title{
Vortex Imaging Using Two-Dimensional Ultrasonic Speckle Correlation
}

\author{
J. Carlson ${ }^{\dagger}$, R. K. Ing ${ }^{\ddagger}$, J. Bercoff ${ }^{\ddagger}$, and M. Tanter ${ }^{\ddagger}$ \\ ${ }^{\dagger}$ EISLAB, Luleå University of Technology, SE-971 87 Luleå, SWEDEN \\ $\ddagger$ Laboratoire Ondes et Acoustique, ESPCI - 10 rue Vauquelin, FR-75231 Paris Cedex 05, FRANCE. \\ E-mail: Johan.Carlson@sm.luth.se
}

\begin{abstract}
In previous work, it has been shown that ultrasonic speckle velocimetry can be used to measure local particle velocities in flows. So far the technique has been applied to monitor stationary processes. In this paper, we show how ultrasonic speckle velocimetry can be used to dynamically map the two-dimensional velocity profiles of vortices caused by an obstacle within a flow. Thanks to the great versatility of our multi-channel system, it is possible to capture as much as $\mathbf{5 0 0 0}$ images per second, thus enabling us to monitor very fast moving processes. To date, two transducer arrays are used to estimate the 2D motion vector of local particles.

We also discuss possible modifications and improvements of the system that could lead to the use of a single array of transducers to dynamically map the vectorial velocity fields of flows.
\end{abstract}

\section{INTRODUCTION}

In this paper we present a technique for imaging vortices caused by an object obstructing a flow. It has previously been shown that time-reversal of acoustic fields can be used to find an average velocity profile of a vortex along the longitudinal axis of the sound beam [1]. It has also been shown that in scattering media it is possible to use ultrasonic speckle velocimetry to resolve local velocities in a flow [2], [5].

If an object obstructs a flow, a vortex pattern will form after the obstacle. This is sometimes used in flow measurement, since the frequency of these vortices is proportional to the flow rate [3]. In other applications, the vortices are the source of vibrations and it is therefore of interest to be able to control the vortex formation. In either case it is of interest to study the time evolution of the vortices. Any technique to do this has to be non-invasive and non-intrusive, because they would otherwise obstruct the flow and affect the vortex pattern.

With our experimental setup, we can obtain up to 5000 speckle images per second [4]. This enables us to monitor faster motions than what was previously possible. In this paper we show experimentally how two-dimensional ultrasonic speckle velocimetry can be used to follow the development of vortices in a flow. In section $\mathrm{V}$, we also discuss other possible experimental setups allowing us to perform this dynamic mapping of $2 \mathrm{D}$ velocities using a single array of transducers.

By using two transducer arrays, mounted at a certain angle to each other, we are able to determine the twodimensional mapping of velocity vectors of the vortices. Previous work used two transducer arrays image a stationary vortex. In this paper we have extended this to image non-stationary motions. This is possible because of the high frame rate of the imaging system.

\section{THEORY}

In this section we briefly describe the principle behind the proposed technique. A more detailed description of this can be found in the paper by Sandrin et al. [4].

\section{A. Speckle Imaging}

To image the vortices in the flow, we use a technique based on the back-scattering of ultrasound from microbubbles suspended in the flow. The linear transducer array emits a plane wave into the medium and then records the back-scattered waves, the B-SCAN image.

Fig. 1 (a) is an example of such a B-SCAN image, coming from a cylindrical cloud of scatterers in a pure acoustic diffusion regime. This is a simulated result, to illustrate the principle. True measurement results are shown in section III-B. Applying the beamforming process [2] to the received image - this consists of delaying and summing parts of the signals from the B-SCAN image corresponding to a certain distance from each of the array elements - we then obtain an image of the scatter cloud. As shown in Fig. 1 (b), the cylindrical shape of the cloud is obvious. The image in Fig. 1 (b) is called a speckle image because it shows the structure that encloses the scatterers, comparable to the one achieved in 
(a)

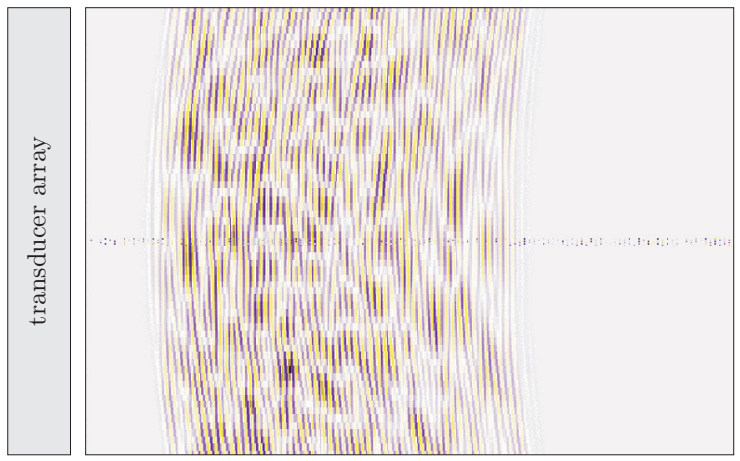

(b)

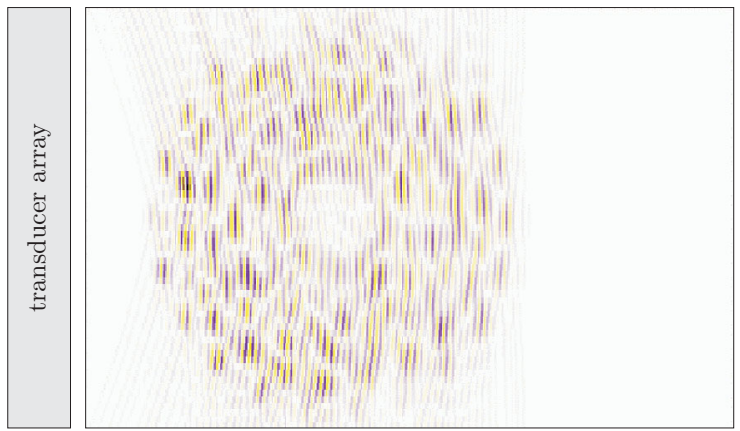

Fig. 1. (a) shows the received B-SCAN image back-scattered from a cloud of scatterers. (b) shows the shape of the cloud after beamforming.

coherent optics. The B-SCAN image, which for each depth shows the interference pattern caused by the scatterers is, however, more directly related to the optical speckle pattern. As is the case in Optics, the ultrasonic speckle location is randomly distributed over the spatial coordinates and its size depends on the aperture of the imaging system. When the scatter cloud moves, slowly and randomly, the corresponding speckle image also moves in a similar way.

By acquiring two speckle images from the same array, closely spaced in time and then cross-correlating line-wise along the depth axis, we can follow how groups of scatters move. The maximum of the cross-correlation function corresponds to the scatter group displacement along the depth axis. The cross-correlation is calculated from short segments of the signal, corresponding to approximately 20 wavelengths. This window is then slided across the signal to obtain local particle displacements. To further increase the resolution of the image we interpolate the cross-correlation function around its maximum using a quadratic function. We can thus observe particle displacements of less than one sample. This corresponds to distances of less than 0.015 $\mathrm{mm}$.

\section{B. Finding $2 D$-Velocities}

Once the particle displacements have been determined for the two transducer arrays separately, we need to combine this to obtain the two-dimensional velocity component. This is done by associating each point in the image obtained with the first array with a point in the image from the second array, corresponding to the same position in the flow. Knowing the angle and the distance between the two arrays we could then calculate the resulting velocity field.

The resulting image was then filtered using a twodimensional low-pass filter. This procedure significantly improved the quality of the final image. In the next section the technique is validated experimentally.

\section{EXPERIMENTS}

This section first describes the setup used in the experiments. The second subsection contains the experimental results.

\section{A. Setup}

In order to experimentally verify the technique, we mounted two 64 element transducer arrays as shown in Fig. 2. A cylindrical obstacle was mounted in front of the transducers and the whole setup was then moved across a big water tank. The water tank was big enough to assure that there were no boundary effects from the walls of the tank. The vortices that formed around the cylindrical obstacle passed the transducer arrays as the setup moved.

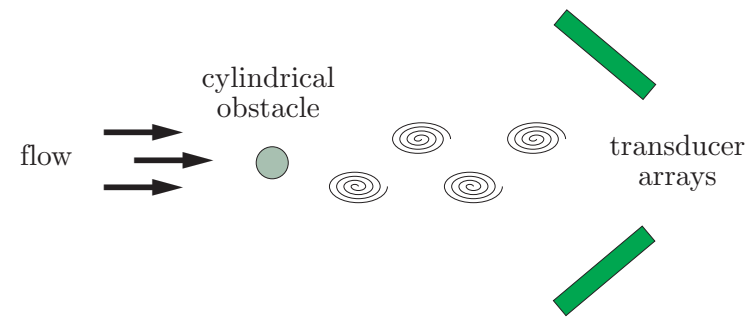

Fig. 2. Experimental setup used in the measurements. Two 64 element transducer arrays mounted at a $130^{\circ}$ angle.

The transducer arrays used in the experiments had a center frequency of 3.5 MHz. Each array consisted of 64 elements separated by $0.834 \mathrm{~mm}$.

The water in the tank was seeded with the medical contrast agent Sonazoid ${ }^{1}$. The contrast agent consists of small

\footnotetext{
${ }^{1}$ Sonazoid is a registered trademark of Nycomed Amersham AS.
} 
micro bubbles, with approximately the same density as water. The algorithm then tracks the movement of these microbubbles.

\section{B. Results}

Fig. 3 shows one image of a pair of vortices passing the two transducer arrays. Notice that the two vortices have different rotation. In the case shown in Fig. 3 the setup with

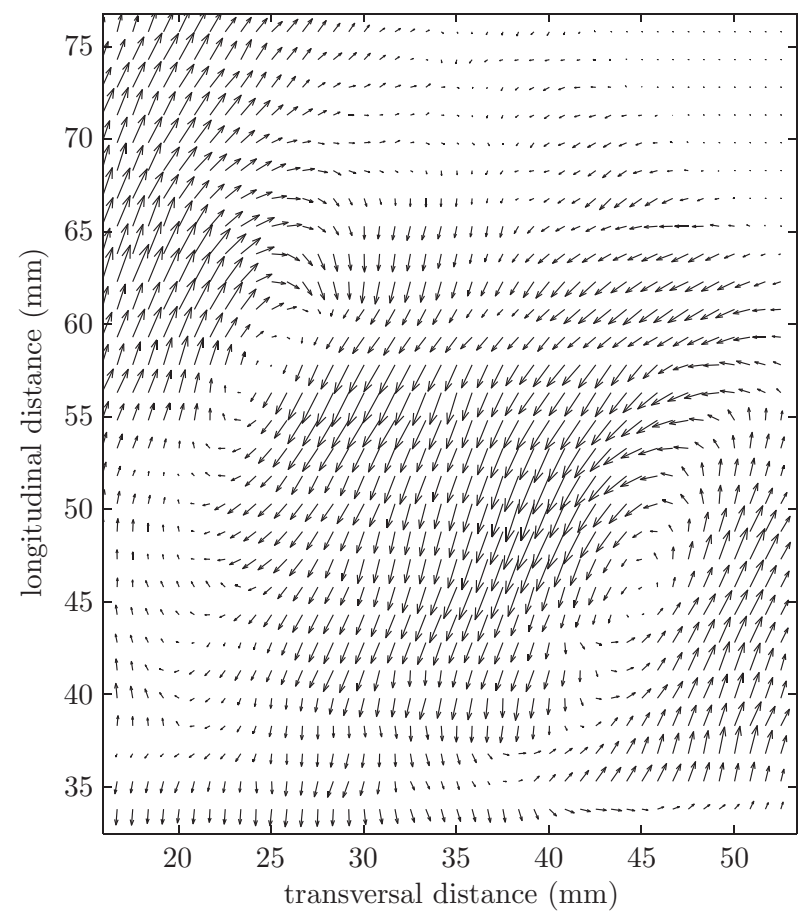

Fig. 3. Two-dimensional velocity vectors of a vortex passing the transducer arrays.

the arrays and the obstacle moved with a velocity of $4 \mathrm{~cm} / \mathrm{s}$. What we see is two vortices with opposite rotation (compare with Fig. 2). The transversal distance (the x-axis), corresponds to the element plane of the first array.

To follow the vortex formation, we recorded images like the one in Fig. 3 with a frame rate of $10 \mathrm{~Hz}$. An animation of these can be found at our web site [6].

\section{Discussion}

In the experiments shown in the previous section, the two transducers were used one at a time, in a very fast transmit/receive scheme. In this way it was possible to assume that the flow was not moving too much. Since the contrast agent micro-bubbles in the flow are assumed to scatter the incident wave spherically, we also tested another transmit/receive scheme. The idea was to use only one of the two arrays to transmit a plane wave, and then record the scat- tered waves with both arrays simultaneously. This would double the maximum achievable frame rate. The problem with this approach was that side lobes from the transmitting array propagated directly to the second array. This signal was obviously much stronger than the scattered waves, and thus saturated the amplifier in the receiver electronics. The same problem occurred if the angle between the two transducer arrays were too small. The optimal angle is $90^{\circ}$ but also in this case, side lobes saturated the receiver. Shielding the arrays from each other with some absorbing material could solve this problem. Another approach is presented in the next section.

Taking full advantage of the available hardware, it is possible to increase the frame rate further, from $2 \mathrm{kHz}$ to $5 \mathrm{kHz}$, thus making it possible to monitor even faster motions. The reason we did not use this higher frame rate here was because the resulting speckle displacements then became too small.

\section{Perspectives}

A new approach consisting of using only one transducer array, but modifying the beamforming process in the receive mode, could be introduced in future work. The use of a single array disturbs the flow characteristics less than the current two-array configuration and should therefore improve the measurement quality. Furthermore, since the cost of a transducer array is fairly high, this would also significantly reduce the total cost of the measurement system.

The new single array approach consists of illuminating the medium with a plane wave and performing the beamforming on the backscattered echoes in the receive mode, on two independent sub-apertures of the array, Fig. 4. This concept is well-known in medical Doppler imaging [7] and here we extend its use to ultra-fast imaging. The measurement of the motion along two different directions $u_{1}$ and $u_{2}$ is here performed by using a plane wave illumination $T_{x}$ from the whole array in the transmit mode, and two lateral sub-apertures $R_{x 1}$ and $R_{x 2}$ in the receive mode. The transmitted plane wave has a $0^{\circ}$ steering angle (in Fig. 4), while the two sub-apertures $R_{x 1}$ and $R_{x 2}$ have different steering angles $\alpha_{0}$ and $\alpha_{1}$. Thus, for each plane wave illumination we calculate two different speckle images ("right" and "left") corresponding to the right and left sub-apertures, respectively.

The vector displacement occurring between two plane wave illuminations is estimated by performing 1D speckle tracking on successive speckle images (i.e. two successive "left" images and two successive "right" images). At a given depth $Z_{0}$ on the $j^{\text {th }}$ line, the $1 \mathrm{D}$ cross-correlation algorithm is then used to estimate the two different time delays, $t_{\alpha 0}$ 


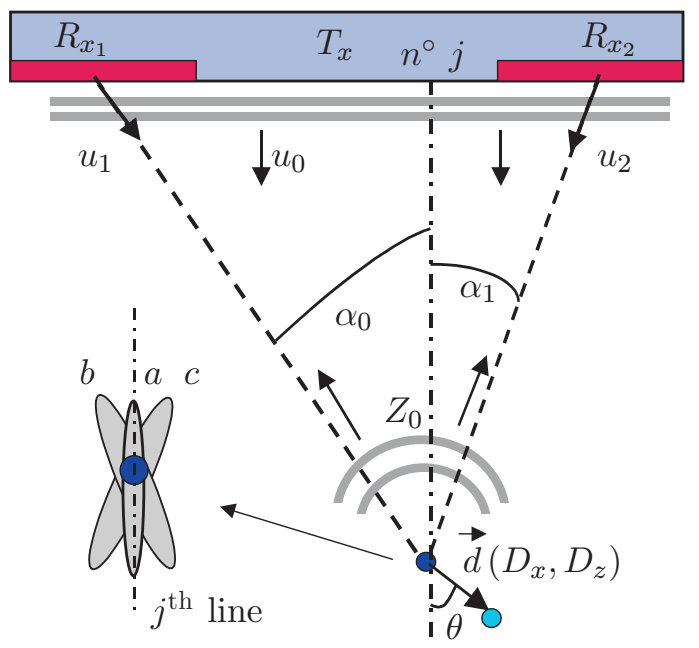

Fig. 4. Left and right sub-apertures giving two different left and right speckle images. The focal spot allowing to process a segment (at depth $Z_{0}$ ) of the $j^{\text {th }}$ line of the image is presented: a) for classical transmit-receive beamforming, b) for left subaperture receiver beamforming, c) for the right sub-aperture receiver beamforming.

and $t_{\alpha 1}$. They correspond to the projections along the two beam directions of the displacement vector of the scatterers contained in the focal region:

$$
\begin{aligned}
t_{\alpha_{0}} & =\frac{d z-d z \cos \alpha_{0}-d x \sin \alpha_{0}}{c} \\
t_{\alpha_{1}} & =\frac{d z+d z \cos \alpha_{1}+d x \sin \alpha_{1}}{c}
\end{aligned}
$$

Note that $\alpha_{0}$ and $\alpha_{1}$ are known as they only depend on geometrical parameters (focal length, sub-aperture position, and sub-aperture width). Thus, the axial and lateral displacements can easily be deduced from the knowledge of the time delays $t_{\alpha 0}$ and $t_{\alpha 1}$ and the steering angle between the two sub-apertures. This angle should be chosen as large as possible in order to maximize the accuracy of the vector displacement estimation. This is because the lateral resolution in the images, determined by the element-pitch of the array, is much lower than the axial resolution. The latter given by the wavelength of the ultrasound and the sampling time of the receiver electronics. Results will be presented in future work.

\section{Conclusions}

Experiments show that ultrasonic speckle velocimetry can be used to monitor fast moving vortices in a flow. In this paper we show how to obtain a two-dimensional velocity field of non-stationary vortices caused by an obstacle obstructing a flow.
The use of plane wave illuminations allows us to achieve ultra-fast frame rates, but also ensure a true instantaneous measurement of the complete $2 \mathrm{D}$ velocity field at each illumination. This is important, since classical transmit-receive focusing techniques used for example in medical Doppler imaging remain limited by the line-by-line acquisition and processing, and therefore introduce a non-uniform measurement grid. This advantage over conventional Doppler imaging becomes even more important in the more academical studies of complex flows in hydrodynamics.

\section{ACKNOWLEDGEMENTS}

The authors would like to express their gratitude towards Dr. Laurent Sandrin for his invaluable help when setting up the experiments, and to professor Mathias Fink for supporting this work. We would also like to thank Nycomed Amersham for providing the contrast agent used in the experiments. This work was made possible by grants from the The Research Council of Norrbotten, Sweden.

\section{REFERENCES}

[1] Roux, P. and Fink, M., "Violation of Time-Reversal Invariance In Acoustics Induced by Vorticity," Europhysics Letters, vol. 32, no. 1, pp. 25-29, 1995.

[2] Sandrin, L., Manneville, S., and Fink, M. "Ultrafast TwoDimensional Speckle Velocimetry: A Tool In Flow Imaging," Applied Physics Letters, vol. 78, no. 8, pp. 1155-1157, 2001.

[3] Lynnworth, L. C. Ultrasonic Measurement For Process Control. Academic Press, 1989.

[4] Sandrin, L., Ing, R. K., and Fink, M. 'Imaging the Intenal Displacement of Groups of Scatterers in Liquid," Proceedings of the 5th European Conference on Underwater Acoustics, (Lyon, France), vol. 1, pp. 673-678, 2000.

[5] Manneville, S., Sandrin, L., and Fink, M. "Investgating a Streched Vortex With Ultrafast 2D Ultrasonic Speckle Velocimetry," Physics of Fluids, vol. 13, no. 6, 2001.

[6] Animation of 2D-Velocity Fields of Vortices, http://www.sm.luth.se/johanc/publications/vortex_movie.avi, October $1,2001$.

[7] Calzolai, M., Capineri, L., Fort, A., Mascotti, L., and Scabia, M. "A Real Time Two-dimensional Pulsed Wave Doppler System," Ultrasound in Medicine and Biology, vol. 26, no. 1, pp. 121-131, 2000. 